\title{
PHOTOMETRIC ANALYSIS IN DETACHED CLOSE BINARIES WITH ECCENTRIC ORBITS. THE CASE OF RX HER
}

\author{
A. GIMÉNEZ \\ Laboratorio de Astrofisica Espacial y Fisica Fundamental (INTA). \\ Apartado 50727. 28080 Madrid, Spain. \\ J. M. GARCÍA and A. BRAVO \\ Departamento de Física, Universidad Politécnica de Madrid \\ Ronda de Valencia, 3. 28012 Madrid, Spain.
}

\begin{abstract}
Absolute dimensions of the close binary RX Her are derived based on a new set of photometric elements obtained with a modified version of EBOP code. The observed properties of both components are in good agreement with theoretical evolutionary models. However the predicted apsidal motion rate is not concordant with the observational evidences.
\end{abstract}

\section{Introduction}

The eclipsing binary system RX Her (HD 170757, $\left.\mathrm{V}=7^{\mathrm{m}} .3, \mathrm{P}=1^{\mathrm{d}} .78, \mathrm{AOV}+\mathrm{B} 9.5 \mathrm{~V}\right)$ is a new case where the phase dispersion around the sccondary minimum may suggest the presence of apsidal motion within the time span of observations.

This is a case similar to those of CW Cep (Clausen and Giménez, 1991) and V 477 Cyg (Giménez and Quintana, 1991) which have been studied recently.

In this communication we present new photometric elements based, for the first time, on photoelectric observations taken in more than one colour. Absolute dimensions of both components have been also calculated and compared with theoretical models. In the last section, apsidal motion is discussed taking into account the available times of minima.

\section{Photometric analysis and absolute dimensions}

Our photometric analysis is based on the UBV observations of RX Her (420 in each colour) that were carried out in 1988 with the $30 \mathrm{~cm}$ telescope of the Ankara University Observatory (Demircan and Derman, 1989).

A modified version of EBOP code has been used to obtain the geometrical elements of RX Her. Besides some other improvements, this version includes the possibility of treat the periastron position as variable. Details of the main changes incorporated to EBOP have been given by Giménez and Díaz-Cordovés in a communication to the $21^{\text {st }}$ IAU General Assembly held in Buenos Aires.

The EBOP code (Etzel, 1981) is faster than other more sophisticated models, and it is specially indicated for detached binaries, like RX Her, where the proximity effects are not very important.

The limb-darkening coefficient for each component were adopted according to the tabulations by Gimenez and Díaz-Cordovés (1991). The mass ratio $q=0.85$ corresponds to the spectroscopic determination by Popper (1959). 
In the iterative process the parameters $i, r_{1}, k$, e $\sin \omega$ and $e \cos \omega$ as well as the magnitude outside eclipses, $\Delta \mathrm{m}$, were alternatively left free to converge towards the final set of values which best reproduce the observed light variations. The final parameters obtained and its error estimates adopted are given in Table 1 . The effective temperature of the hotter component, $T_{1}$, was calculated from the standard uvby- $\mathrm{H} \beta$ colour indices taken from Hilditch and Hill (1975).

Although the eccentricity that we found is roughly the same than Jeffreys's (1980) value, the relative radii of the components and the orbital inclination differ significantly from our determinations. Jeffreys's analysis is based on a single $\mathrm{V}$ light curve solved with the computer model WINK.

TABLE 1. Photometric elements of $\mathrm{RX}$ Her derived with EBOP

\begin{tabular}{|c|c|c|c|c|}
\hline & $\mathrm{U}$ & B & V & \\
\hline $\mathrm{J}_{2} / \mathrm{J}_{1}$ & 0.746 & 0.818 & 0.842 & \multirow{5}{*}{$\begin{array}{l}0.240 \pm 0.003 \\
0.180 \pm 0.002\end{array}$} \\
\hline$r_{1}$ & 0.243 & 0.237 & 0.240 & \\
\hline$r_{2}$ & 0.182 & 0.178 & 0.180 & \\
\hline$u_{1}$ & 0.59 & 0.53 & 0.44 & \\
\hline $\mathrm{u}_{2}$ & 0.63 & 0.56 & 0.47 & \\
\hline$i^{*}\left({ }^{\circ}\right)$ & 86.0 & 86.4 & 86.8 & \multirow[t]{3}{*}{$86.4 \pm 0.3$} \\
\hline e $\cos \omega$ & & & 15 & \\
\hline e $\sin \omega$ & & & 250 & \\
\hline & 0.92 & 0.77 & 0.65 & \multirow{6}{*}{$\begin{array}{c}0.025 \pm 0.015 \\
93 \pm 3\end{array}$} \\
\hline $\begin{array}{l}y_{2} \\
\mathrm{mag} \quad(\text { Phase }=.25)\end{array}$ & $\begin{array}{l}0.94 \\
-0.629\end{array}$ & $\begin{array}{c}0.83 \\
-0.888\end{array}$ & 0.70 & \\
\hline $\mathrm{e}^{\mathrm{mag}(\text { Pnase }=.25)}$ & -0.029 & -0.000 & -0.832 & \\
\hline$\omega\left({ }^{\circ}\right)$ & & & & \\
\hline $\begin{array}{l}\mathrm{L}_{1} \\
\mathrm{~L}_{2}\end{array}$ & $\begin{array}{l}0.712 \\
0.287\end{array}$ & $\begin{array}{l}0.690 \\
0.310\end{array}$ & $\begin{array}{l}0.685 \\
0.315\end{array}$ & \\
\hline$\sigma(\mathrm{mag})$ & 0.0250 & 0.0367 & 0.0218 & \\
\hline
\end{tabular}

Absolute dimensions given in Table 2 have been calculated using the spectroscopic data established by Popper $\left(\mathrm{K}_{1}=138 \mathrm{~km} / \mathrm{s}, \mathrm{K}_{2}=163 \mathrm{~km} / \mathrm{s}\right)$ and the photometric elements listed in Table 1. We have compared the observed properties of RX Her with the evolutionary models computed by Claret and Gimenez (1989) carrying both components to the $\log g-\log T_{e}$ diagram. Both stars are located halfway between the ZAMS and the TAMS and fall roughly on the same isochrone. Ages of $2.5 \times 10^{8} \mathrm{yr}$ are found for the two stars. This is slightly larger than the synchronization time scale, but smaller than the circularization time scale.

TABLE 2. Absolute dimensions of RX Her

\begin{tabular}{|l|cc|}
\hline & Component A & Component B \\
\hline $\mathrm{m} / \mathrm{M}_{\odot}$ & $2.74 \pm 0.15$ & $2.32 \pm 0.12$ \\
$\mathrm{R} / \mathrm{R}_{\odot} \mathrm{T}$ & $2.56 \pm 0.05$ & $1.92 \pm 0.04$ \\
$\log \mathrm{T}$ (c.g.s.) & $4.045 \pm 0.007$ & $4.004 \pm 0.005$ \\
$\log \mathrm{g}$ (cos & $4.06 \pm 0.04$ & $4.24 \pm 0.04$ \\
$\log / \mathrm{L}_{\odot}$ & $1.95 \pm 0.05$ & $1.54 \pm 0.04$ \\
\hline
\end{tabular}


On the other hand, the observed temperatures coincide with the theoretical values predicted by the models for a star with the mass and $\log \mathrm{g}$ observed. This denotes a good agreement with the chemical solar composition $(X, Z)=(0.70,0.02)$.

\section{Apsidal motion}

The newtonian contribution to the apsidal motion can be calculated from the estimated dimensions of the stars. The rotational velocities of the primary $(80 \mathrm{~km} / \mathrm{s})$ and the secondary $(70 \mathrm{~km} / \mathrm{s})$ components have been taken from Tan (1988), while the internal structure constants were adopted from the theoretical models by Claret and Gimenez (1989). This leads to a value of $\dot{\omega}_{\mathrm{N}}=0.027^{\circ} /$ cycle.

On the other hand, using the absolute dimensions in Table 2 and the equation for the relativistic contribution to apsidal motion we obtain a value of $\dot{\omega}_{\mathrm{R}}=0.0012 \%$ cycle, and thus a total rate of $\dot{\omega}=0.028 \%$ cycle.

The expressions given by Gimenez (1985) allow us to calculate the difference $\triangle P$ between the linear periods of the primary, $\mathrm{P}_{1}$, and of the secondary, $\mathrm{P}_{2}$, which results from the previous $\dot{\omega}$. We have obtained the value $(\triangle P)_{\text {pred }}=(6.9 \pm 5.1) \times 10^{-6} \mathrm{~d}$. However, the study of 29 available photoelectric time of minima yields two identical values, within the involved errors for $P_{1}$ and $P_{2}$. In other words, $(\triangle \mathrm{P})_{\text {obs }}=(0.0 \pm 5.4) \times 10^{-7} \mathrm{~d}$.

Those results suggest that this could be another example of close binary in disagreement with theoretical predictions. The confirmation of this fact has to wait for a more precise determination of the orbital eccentricity which is needed to reduce significantly the error in $(\triangle P)_{\text {pred. }}$

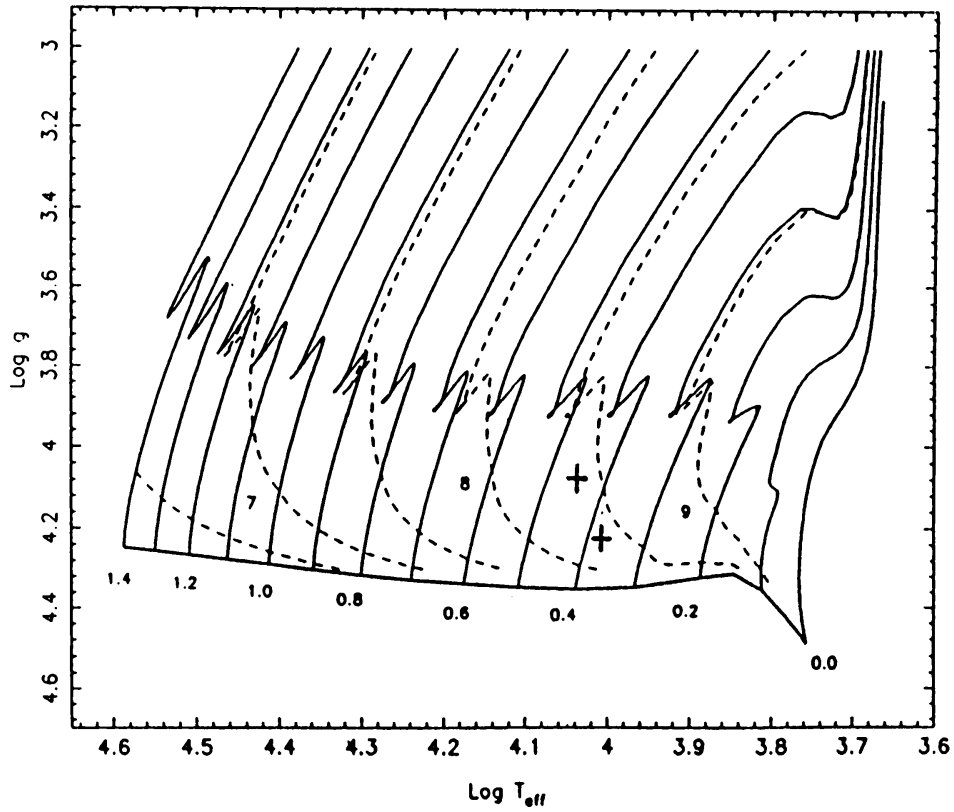

Figure 1. Components of RX Her on the diagram $\log g-\log T_{e}$. The evolutionary tracks have been taken from the models by Claret and Gimenez (1989) for a initial chemical composition $(\mathrm{X}, \mathrm{Z})=(0.70,0.02)$. 


\section{Acknowledgements}

This work has been supported by the Spanish DGICYT through project PB87-0235.

\section{References}

Claret, A. and Giménez, A. (1989) Astron. Astrophys. Suppl. 81, 1

Demircan, O. and Derman, E. (1989), IBVS No. 3403

Etzel, P.B. (1981) in Photometric and Spectroscopic Binary Systems, eds. E.B. Carling and Z. Kopal, D. Reidel Pub. Co., Dordrecht, p. 111.

Giménez, A. (1985) Astrophys. J. 279, 405.

Gimenez, A. and Clausen, J.V. (1991), Astron. Astrophys. 241, 98

Giménez, A. and Díaz Cordovés, J. (1991), submited to Astron. Astrophys.

Giménez, A. and Quintana, J.M. (1991), submited to Astron. Astrophys.

Hilditch, R.W. and Hill, G. (1975), Mem. R. Astron. Soc. 79, 123

Jeffreys, K.W. (1980), Astron. Astrophys. Suppl. 42, 285

Popper, D.M. (1959), Astrophys. J. Suppl. 29, 43

Tan, H-S. (1988), Acta Astron. Sinica 30, 136 